\title{
THE KERNEL-TRACE APPROACH TO RIGHT CONGRUENCES ON AN INVERSE SEMIGROUP
}

\author{
MARIO PETRICH AND STUART RANKIN
}

\begin{abstract}
A kernel-trace description of right congruences on an inverse semigroup is developed. It is shown that the trace mapping is a complete $\cap$ homomorphism but not a $V$-homomorphism. However, the trace classes are intervals in the complete lattice of right congruences. In contrast, each kernel class has a maximum element, namely the principal right congruence on the kernel, but in general there is no minimum element in a kernel class. The kernel mapping preserves neither intersections nor joins.

The set of axioms presented in [7] for right kernel systems is reviewed. A new set of axioms is obtained as a consequence of the fact that a right congruence is the intersection of the principal right congruences on the idempotent classes.

Finally, it is shown that even though a congruence on a regular semigroup is the intersection of the principal congruences on the idempotent classes, the situation is not the same for right congruences on a regular semigroup. Right congruences on a regular, even orthodox, semigroup are not, in general, determined by their idempotent classes.
\end{abstract}

\section{INTRODUCTION AND SUMMARY}

In 1953, Wagner [14] proved that every congruence on an inverse semigroup is completely determined by its idempotent classes. The following year, Preston [12] characterized those collections of subsets which arise as the idempotent classes of some congruence, and referred to such collections as kernel normal systems. Some twenty years later, in [13] Scheiblich introduced the kernel-trace description for congruences on an inverse semigroup. By this time, it was well known that every one-sided congruence on an inverse semigroup was completely determined by its idempotent classes. In 1974, Meakin [7] provided a characterization for right (left) kernel systems, the one-sided analogue of the kernel normal systems of Preston. He also considered the trace of a right congruence, and showed that every congruence relation on the semilattice of idempotents occurs as the trace of some right congruence on the inverse semigroup itself. As well, he established that every trace class of right congruences has a greatest and a least element, and classified those inverse semigroups for which the trace class of the equality trace is smallest possible, that is, consisting of $\varepsilon$ and $\mathscr{L}$.

We present the kernel-trace approach to right congruences on an inverse semigroup. It is shown how to contruct a right congruence upon being given its ker-

Received by the editors October 5,1989 and, in revised form, March 8, 1990.

1980 Mathematics Subject Classification (1985 Revision). Primary 20M10.

Key words and phrases. Right congruences, inverse semigroup, regular semigroup, kernel, trace, right kernel system, Clifford semigroup. 
nel and its trace. Right kernels are characterized, and necessary and sufficient conditions are given in order that a right kernel and a trace form a right congruence pair, that is to say, determine a right congruence having as its kernel and trace the given right kernel and trace, respectively. The notion of a pseudo right congruence pair turns out to be useful. A pseudo right congruence pair consists of a full subsemigroup and a trace which together contain the information to determine a right congruence having as its trace the given trace and its kernel contained in the given subsemigroup, which is then referred to as a pseudo kernel of the right congruence. Necessary and sufficient conditions that a subsemigroup must satisfy in order that it be a pseudo kernel for a given right congruence are established.

The kernel-trace description is utilized to describe all right congruences on Clifford semigroups. Since Nico [8] has described all right congruences on the bicyclic semigroup (see Duchamp [2] as well) and we on a Brandt semigroup [10], we have a rich supply of examples.

For any inverse semigroup $S$ with semilattice of idempotents $E$, we obtain mappings from the lattice of right congruences on $S$ to the lattice of congruences on $E$ and to the lattice of full subsemigroups of $S$ by sending a congruence to its trace and its kernel, respectively. It is shown that the trace mapping is a complete $\mathrm{n}$-homomorphism but not a $\mathrm{V}$-homomorphism. However, the trace classes are intervals in the complete lattice of right congruences. In contrast, each kernel class has a maximum element, namely the principal right congruence on the kernel, but in general there is no minimum element in a kernel class. The kernel mapping preserves neither intersections nor joins.

The set of axioms presented in [7] for right kernel systems is reviewed. A new set of axioms is obtained as a consequence of the fact that a right congruence is the intersection of the principal right congruences on the idempotent classes.

Finally, it is shown that even though a congruence on a regular semigroup is the intersection of the principal congruences on the idempotent classes, the situation is not the same for right congruences on a regular semigroup. Right congruences on a regular, even orthodox, semigroup are not, in general, determined by their idempotent classes.

\section{Preliminaries}

Recall that for any subset $X$ of a semigroup $S$, the principal right congruence $\mathrm{P}_{X}$ on $S$ is the greatest right congruence on $S$ saturating $X$. If $S$ is an inverse semigroup, then $a \mathrm{P}_{X} b$ if and only if for all $u \in S, a u \in X \Leftrightarrow b u \in X$.

Throughout the paper, $S$ shall denote an inverse semigroup, unless noted otherwise. For any subset $A$ of $S, E(A)$ shall denote the set of idempotents of $S$ contained in $A$, with $E(S)$ abbreviated to $E$. The lattice of right congruences on $S$ and the lattice of congruences on $E$ shall be denoted by $\mathscr{R} \mathscr{C}(S)$ and $\mathscr{C}(E)$, respectively. The identity and universal relations on any set shall be denoted by $\varepsilon$ and $\omega$, respectively. In general, we shall follow [9] for notation and terminology.

\section{THE KERNEL-TRACE DESCRIPTION OF A RIGHT CONGRUENCE}

After the necessary definitions, we present the principal result of the paper: the kernel-trace description of right congruences on an inverse semigroup. 
Definition 3.1. For any right congruence $\rho$ on $S, \operatorname{ker} \rho=\bigcup_{e \in E} e \rho$ is the kernel of $\rho$ and $\operatorname{tr} \rho=\left.\rho\right|_{E}$ is the trace of $\rho$.

We shall show in this section that $\rho$ is determined by the pair $(\operatorname{ker} \rho, \operatorname{tr} \rho)$.

Lemma 3.2. For any right congruence $\rho$ on $S, \operatorname{ker} \rho$ is a full subsemigroup of $S$ and $\operatorname{tr} \rho$ is a congruence on $E$.

Proof. If $a \rho e$ and $b \rho f$, where $e, f \in E$, then

$$
a b \rho e b=b b^{-1} e b \rho f b^{-1} e b .
$$

Definition 3.3. Let $K$ be a full subsemigroup of $S$ and $\tau$ a congruence on $E$ satisfying the following two conditions.

(i) For all $a \in S, b \in K, a \geq b$ and $a a^{-1} \tau b b^{-1}$ imply that $a \in K$.

(ii) For all $a \in K, e, f \in E, e \tau f$ implies that $a e a^{-1} \tau a f a^{-1}$.

Then $(K, \tau)$ is said to be a pseudo right congruence pair for $S$. If, in addition, the condition

(iii) For every $a \in K$, there exists $b \in S$ with $a \geq b, a a^{-1} \tau b b^{-1}$ and $b^{-1} \in K$

is satisfied, then $(K, \tau)$ is said to be a right congruence pair for $S$. For a pseudo right congruence pair $(K, \tau)$, define a relation $\rho_{(K, \tau)}$ on $S$ by

$$
a \rho_{(K, \tau)} b \Leftrightarrow a b^{-1}, b a^{-1} \in K, \quad a b^{-1} b a^{-1} \tau a a^{-1}, \quad b a^{-1} a b^{-1} \tau b b^{-1} .
$$

We remark that, unlike the case for two-sided congruences on an inverse semigroup, the explicit symmetry of the definition is necessary. For if $K$ is not an inverse subsemigroup of $S$, then there exists $a \in K$ for which $a^{-1} \notin K$. Let $b=a^{-1} a$. Then $a b^{-1} \in K, a b^{-1} b a^{-1}=a a^{-1}$ and $b a^{-1} a b^{-1}=b b^{-1}$ but $b a^{-1} \notin K$.

Proposition 3.4. If $(K, \tau)$ is a pseudo right congruence pair for $S$, then $\rho_{(K, \tau)}$ is a right congruence on $S$ which saturates $K$ with

(1) $\operatorname{ker} \rho_{(K, \tau)}=\left\{a \in K \mid\right.$ there exists $b$ with $\left.a \geq b, a a^{-1} \tau b b^{-1}, b^{-1} \in K\right\}$

and trace equal to $\tau$. Moreover, if $\left(K_{1}, \tau_{1}\right)$ and $\left(K_{2}, \tau_{2}\right)$ are pseudo right congruence pairs for $S$ with $K_{1} \subseteq K_{2}$ and $\tau_{1} \subseteq \tau_{2}$, then $\rho_{\left(K_{1}, \tau_{1}\right)} \subseteq \rho_{\left(K_{2}, \tau_{2}\right)}$.

Proof. Let $(K, \tau)$ be a pseudo right congruence pair and set $\rho=\rho_{(K, \tau)}$. Since $K$ is full it follows that $\rho$ is reflexive. Obviously, $\rho$ is symmetric. We defer the verification that $\rho$ is transitive until after we have established that $\rho$ is right compatible. Assume now that $a \rho b$ and let $c \in S$. We have

$$
(a c)(b c)^{-1}=a c c^{-1} b^{-1}=\left(a b^{-1}\right)\left(b c c^{-1} b^{-1}\right)
$$

and since $a b^{-1} \in K$ and $b c c^{-1} b^{-1} \in E \subseteq K$, it follows that $(a c)(b c)^{-1} \in K$. Similarly,

$$
(b c)(a c)^{-1}=b c c^{-1} a^{-1}=\left(b c c^{-1} b^{-1}\right)\left(b a^{-1}\right) \in K .
$$

Next, we have

$$
\begin{aligned}
(a c)(b c)^{-1}(b c)(a c)^{-1} & =a c c^{-1} b^{-1} b c c^{-1} a^{-1}=a b^{-1} b c c^{-1} a^{-1} \\
& =\left(a b^{-1} b a^{-1}\right)\left(a c c^{-1} a^{-1}\right) \tau\left(a a^{-1}\right)\left(a c c^{-1} a^{-1}\right) \\
& =(a c)(a c)^{-1}
\end{aligned}
$$


By symmetry, it follows that

$$
(b c)(a c)^{-1}(a c)(b c)^{-1} \tau(b c)(b c)^{-1}
$$

whence $a c \rho b c$. Thus $\rho$ is a right compatible relation.

Let $a \rho b$ and $b \in K$. Then by the definition of $\rho$, we have

$$
a b^{-1} b a^{-1} \tau a a^{-1} \text { and }\left(a b^{-1}\right) b \in K \text {. }
$$

Now by Definition 3.3(i), $a \geq a b^{-1} b \in K$ and $a a^{-1} \tau\left(a b^{-1} b\right)\left(a b^{-1} b\right)^{-1}$ implies that $a \in K$. Thus $\rho$ saturates $K$. Now, suppose that $a \rho b$ and $b \rho c$. Then $a c^{-1} \rho b c^{-1}$ and $b c^{-1} \rho c c^{-1}$. Since $c c^{-1} \in K$ and $b c^{-1} \rho c c^{-1}$, we obtain $b c^{-1} \in K$ and subsequently $a c^{-1} \in K$. Similarly, $c a^{-1} \rho b a^{-1}$ and $b a^{-1} \rho a a^{-1}$ by saturation yield $c a^{-1} \in K$. Next, from $c b^{-1} b c^{-1} \tau c c^{-1}$ we obtain

$$
\left(c b^{-1} b c^{-1}\right)\left(c a^{-1} a c^{-1}\right) \tau\left(c c^{-1}\right)\left(c a^{-1} a c^{-1}\right)=c a^{-1} a c^{-1},
$$

while from $b a^{-1} a b^{-1} \tau b b^{-1}$ and $c b^{-1} \in K$, Definition 3.3(ii) yields

$$
\begin{aligned}
\left(c b^{-1} b c^{-1}\right)\left(c a^{-1} a c^{-1}\right) & =\left(c b^{-1}\right)\left(b a^{-1} a b^{-1}\right)\left(c b^{-1}\right)^{-1} \tau\left(c b^{-1}\right)\left(b b^{-1}\right)\left(c b^{-1}\right)^{-1} \\
& =c b^{-1} b c^{-1} .
\end{aligned}
$$

Thus we obtain

$$
c a^{-1} a c^{-1} \tau\left(c b^{-1} b c^{-1}\right)\left(c a^{-1} a c^{-1}\right) \tau c b^{-1} b c^{-1} \tau c c^{-1} .
$$

In a similar manner, we obtain from Definition 3.3(ii) applied to

$$
b c^{-1} c b^{-1} \tau b b^{-1}
$$

and $a b^{-1} \in K$ that $\left(a b^{-1} b a^{-1}\right)\left(a c^{-1} c a^{-1}\right) \tau a b^{-1} b a^{-1}$, whence

$$
a c^{-1} c a^{-1}=\left(a a^{-1}\right)\left(a c^{-1} c a^{-1}\right) \tau\left(a b^{-1} b a^{-1}\right)\left(a c^{-1} c a^{-1}\right) \tau a b^{-1} b a^{-1} \tau a a^{-1} \text {. }
$$

Thus $a \rho c$ as required. We have now demonstrated that $\rho$ is a right congruence which saturates $K$.

It is apparent that for $e, f \in E, e \rho f$ if and only if $e \tau f$ whence $\operatorname{tr} \rho=\tau$. We let

$$
L=\left\{a \in K \mid \text { there exists } b \text { such that } a \geq b, b^{-1} \in K, a a^{-1} \tau b b^{-1}\right\}
$$

and show that $\operatorname{ker} \rho=L$. Let $a \in L$ so that for some $b \leq a$, we have $b^{-1} \in K$ and $a a^{-1} \tau b b^{-1}$. Let $e=b^{-1} b$. We show that $a \rho e$. Since $a \in L \subseteq K$, we have $a e \in K, e a^{-1}=b^{-1} \in K$, aea $a^{-1}=b b^{-1} \tau a a^{-1}$ and $e a^{-1} a e=e$ whence by definition of $\rho, a \rho e$. Thus $a \in \operatorname{ker} \rho$ and so $L \subseteq \operatorname{ker} \rho$. Conversely, let $a \in \operatorname{ker} \rho$. Then $a \rho e$ for some $e \in E$. Let $b=a e$ so that $b \leq a$ and as well, $a a^{-1} \rho e a^{-1}$ whence $b^{-1}=e a^{-1} \in K$. Now compute $b b^{-1}=a e a^{-1} \rho e a^{-1} \rho a a^{-1}$ so that $a \in L$. Thus $\operatorname{ker} \rho \subseteq L$.

The last assertion of the proposition is immediate.

Theorem 3.5. If $(K, \tau)$ is a right congruence pair for $S$, then $\rho_{(K, \tau)}$ is a right congruence on $S$ with kernel $K$ and trace $\tau$. Conversely, if $\rho$ is a right congruence on $S$, then ( $\operatorname{ker} \rho, \operatorname{tr} \rho$ ) is a right congruence pair for $S$ and $\rho=\rho_{(\operatorname{ker} \rho, \operatorname{tr} \rho)}$.

Proof. Let $(K, \tau)$ be a right congruence pair and let $\rho=\rho_{(K, \tau)}$. Then by Proposition 3.4, $\rho$ is a right congruence with trace equal to $\tau$ and $\operatorname{ker} \rho$ as in (1). Thus $\operatorname{ker} \rho \subseteq K$. Now let $a \in K$. Then by Definition 3.3(iii), there 
exists $b \in S$ with $b \leq a, b^{-1} \in K$ and $b b^{-1} \tau a a^{-1}$ whence $a \in \operatorname{ker} \rho$ by Proposition 3.4. Thus $K=\operatorname{ker} \rho$.

Conversely, let $\rho$ be a right congruence on $S$ and let $K=\operatorname{ker} \rho, \tau=\operatorname{tr} \rho$. Then $K$ is a full subsemigroup of $S$ by Lemma 3.2 and $\tau$ is a congruence on $E$. Let $a \geq b \in K$ and suppose that $a a^{-1} \tau b b^{-1}$. Then $b=b b^{-1} a$ and $a a^{-1} \tau b b^{-1}$ whence $a \rho b b^{-1} a$ and so $b=b b^{-1} a \rho a$ and hence $a \in b \rho \subseteq$ $\operatorname{ker} \rho=K$. Thus Definition 3.3(i) holds for $(K, \tau)$. Next, let $a \in K$ and suppose that $e \tau f$ for some $e, f \in E$. Since $a \in K$ we have $a \rho e^{\prime}$ for some $e^{\prime} \in E$. Then ae $\rho e^{\prime} e=e e^{\prime} \rho f e^{\prime}=e^{\prime} f \rho$ af and so $a e a^{-1} \tau a f a^{-1}$. Thus Definition 3.3(ii) holds. For $a \in K$ there exists $e \in E$ with $a \rho e$. Since $a \rho e a^{-1} a$, we may assume that $e \leq a^{-1} a$, whence $b=a e \leq a$. Also from $a \rho e$ we obtain $b \rho e \rho a$ whence $b b^{-1} \rho a a^{-1}$. Moreover, $a a^{-1} \rho e a^{-1}$ and so $b^{-1}=e a^{-1} \in K$. Thus Definition 3.3(iii) is satisfied and so $(K, \tau)$ is a right congruence pair.

It remains to prove that $\rho=\rho_{(K, \tau)}$. Let $a \rho b$. Then we have $a b^{-1} \rho b b^{-1}$ $\in E$ and $b a^{-1} \rho a a^{-1} \in E$ whence $a b^{-1}, b a^{-1} \in K$ and

$$
a b^{-1} b a^{-1} \rho b b^{-1} b a^{-1}=b a^{-1} \rho a a^{-1} \text {. }
$$

Thus $a b^{-1} b a^{-1} \tau a a^{-1}$. Similarly, we have $b a^{-1} a b^{-1} \tau b b^{-1}$ and so $a \rho_{(K, \tau)} b$. Therefore $\rho \subseteq \rho_{(K, \tau)}$. Now let $a \rho_{(K, \tau)} b$. Then $a b^{-1}, b a^{-1}$ $\in K$ and $a b^{-1} b a^{-1} \tau a a^{-1}, b a^{-1} a b^{-1} \tau b b^{-1}$. We have $a b^{-1} b \rho a$ and $b a^{-1} a \rho b$. As well, there exist $e, f \in E$ with $a b^{-1} \rho e$ and $b a^{-1} \rho f$. Consequently, $a b^{-1} b \rho e b=b e^{\prime}$ where $e^{\prime}=b^{-1} e b$ and $b a^{-1} a \rho f a=$ $a f^{\prime}$ where $f^{\prime}=a^{-1} f a$. Thus $a \rho a b^{-1} b \rho e b \rho b e^{\prime}$ yields $a e^{\prime} \rho a$. Then $b \rho b a^{-1} a \rho a f^{\prime} \rho a e^{\prime} f^{\prime}$ and so $b e^{\prime} \rho b$. It follows that $a \rho b e^{\prime} \rho b$. We have $\rho_{(K, \tau)} \subseteq \rho$ and so equality prevails.

Definition 3.6. Given a right congruence $\rho$, a full subsemigroup $K$ of $S$ for which $(K, \operatorname{tr} \rho)$ is a pseudo right congruence pair for $S$ such that $\rho=\rho_{(K, \tau)}$ shall be called a pseudo right kernel of $\rho$.

We observe that necessarily each pseudo kernel of $\rho$ contains $\operatorname{ker} \rho$ and is saturated by $\rho$. Furthermore, by Definition 3.3(ii), it consists of elements of $S$ which normalize the trace of $\rho$.

Definition 3.7. For any $\tau \in \mathscr{C}(E)$, let $N^{\tau}$ denote the normalizer in $S$ of $\tau$, that is,

$$
N^{\tau}=\left\{a \in S \mid e \tau f \text { implies that } a e a^{-1} \tau a f a^{-1}\right\} .
$$

It is evident that for any congruence $\tau$ on $E, N^{\tau}$ is a full subsemigroup of $S$.

Proposition 3.8. Let $\rho$ be a right congruence on $S$ with kernel $K$ and trace $\tau$. $A$ subsemigroup $T$ of $S$ is a pseudo right kernel for $\rho$ if and only if $K \subseteq T \subseteq N^{\tau}$, $T$ is saturated by $\rho$, and for all $a \in S, a \in T \backslash K$ implies $a^{-1} \notin T$.

Proof. Let $T$ be a pseudo right kernel for $\rho$. Then, as observed above, $K \subseteq$ $T \subseteq N^{\tau}$ and $\rho$ saturates $T$. Let $a \in T$ and suppose that $a^{-1} \in T$. By Proposition 3.4, taking $b=a$, we see that $a \in \operatorname{ker} \rho=K$. Thus $a \in T \backslash K$ implies that $a^{-1} \notin T$.

Conversely, let $T$ be a subsemigroup of $S$ satisfying the conditions of the proposition. Let $a \geq b \in T$ and $a a^{-1} \rho b b^{-1}$. Then $a \rho b b^{-1} a=b \in T$ 
and then since $T$ is saturated by $\rho$, we have $a \in T$. Thus Definition 3.3(i) holds. Definition 3.3(ii) is satisfied since $T \subseteq N^{\tau}$ and so $(T, \tau)$ is a pseudo right congruence pair. Now by Theorem 3.5 and Proposition 3.4, respectively, we have $\rho=\rho_{(K, \tau)} \subseteq \rho_{(T, \tau)}$. Let $a \rho_{(T, \tau)} b$. Then by definition, we have $a b^{-1} \in T, b a^{-1} \in T, a b^{-1}\left(a b^{-1}\right)^{-1} \tau a a^{-1}$ and $b a^{-1}\left(b a^{-1}\right)^{-1} \tau b b^{-1}$. If $a b^{-1} \in T \backslash K$, then $b a^{-1}=\left(a b^{-1}\right)^{-1} \notin T$, a contradiction. Thus $a b^{-1} \in K$ and similarly $b a^{-1} \in K$. By definition, we have $a \rho_{(K, \tau)} b$, that is, $a \rho b$. Thus $\rho_{(T, \tau)} \subseteq \rho$ and so we have equality.

Proposition 3.9. Let $\left\{\left(K_{\alpha}, \tau_{\alpha}\right) \mid \alpha \in A\right\}$ be a collection of pseudo right congruence pairs, and let $K=\bigcap_{\alpha \in A} K_{\alpha}, \tau=\bigcap_{\alpha \in A} \tau_{\alpha}$. Then $(K, \tau)$ is a pseudo right congruence pair and $\rho_{(K, \tau)}=\bigcap_{\alpha \in A} \rho_{\left(K_{\alpha}, \tau_{\alpha}\right)}$.

Proof. This follows by direct computation.

We conclude this section with a description of the (pseudo) right congruence pairs for $S$ for which the trace is extremal.

Lemma 3.10. Let $K$ be a full subsemigroup of $S$.

(i) $(K, \varepsilon)$ is a pseudo right congruence pair for $S$, while $(K, \varepsilon)$ is a right congruence pair if and only if $K$ is an inverse semigroup.

(ii) $(K, \omega)$ is a pseudo right congruence pair for $S$ if and only if $K$ is closed, while $(K, \omega)$ is a right congruence pair for $S$ if and only if $K$ is a closed inverse subsemigroup of $S$.

Proof. (i) To verify that Definition 3.3(i) holds for $(K, \varepsilon)$, let $a \geq b \in K$ and $a a^{-1}=b b^{-1}$. Then $a=a a^{-1} a=b b^{-1} a=b \in K$. Since Definition 3.3(ii) obviously holds, it follows that $(K, \varepsilon)$ is a pseudo right congruence pair for $S$. Suppose now that $(K, \varepsilon)$ is actually a right congruence pair. Then for each $a \in K$ there exists $b \leq a$ such that $b^{-1} \in K$ and $a a^{-1}=b b^{-1}$ by Proposition 3.4. Thus $a^{-1}=a^{-1} a a^{-1}=a^{-1} b b^{-1} \in E b^{-1} \subseteq K$ since $a^{-1} b \in E$. It follows therefore that $K$ is an inverse semigroup. Conversely, if $K$ is an inverse semigroup, then for any $a \in K$, we may apply Proposition 3.4 with $b=a$ to see that $a \in \operatorname{ker} \rho_{(K, \varepsilon)}$ whence $(K, \varepsilon)$ is a right congruence pair.

(ii) From the definition, $(K, \omega)$ is a pseudo right congruence pair if and only if $K$ is closed. Suppose that $K$ is closed. Then Proposition 3.4 immediately establishes that $\operatorname{ker} \rho_{(K, \omega)}=\left\{a \in K \mid a^{-1} \in K\right\}$. Thus $(K, \omega)$ is a right congruence pair if and only if $K$ is a closed inverse subsemigroup of $S$.

\section{KERNELS AND TRACES}

The kernel-trace approach to right congruences would have little advantage over the kernel system approach if one could not identify those full subsemigroups of $S$ which appear as kernels of right congruences, and those congruences on $E$ which appear as traces of right congruences. As we remarked at the outset, every congruence on $E$ is the trace of some right congruence on $S$, and there is a greatest and a least right congruence having a specified trace. We present a kernel-trace description of the greatest and the least right congruences having a given trace.

Proposition 4.1. Let $\tau \in \mathscr{C}(E)$. Then $\left(N^{\tau}, \tau\right)$ is a pseudo right congruence pair for $S$ and $\rho_{\left(N^{\tau}, \tau\right)}$ is the greatest right congruence on $S$ with trace $\tau$. 
Proof. It is evident that $N^{\tau}$ is a full subsemigroup of $S$ and that Definition 3.3(ii) is satisfied. In order to verify Definition 3.3(i), let $a \geq b \in N^{\tau}$ and $a a^{-1} \tau b b^{-1}$. We must show that $a \in N^{\tau}$. Let $e \tau f$. It follows from $a \geq b$ that $b^{-1} a$ is an idempotent whence

$$
\begin{aligned}
a e a^{-1} & =\left(a a^{-1}\right)\left(a e a^{-1}\right) \tau\left(b b^{-1}\right)\left(a e a^{-1}\right) \\
& =b e b^{-1} a a^{-1} \tau b e b^{-1} b b^{-1}=b e b^{-1} .
\end{aligned}
$$

Similarly, $a f a^{-1} \tau b f b^{-1}$. Moreover, $b e b^{-1} \tau b f b^{-1}$ since $b \in N^{\tau}$. Thus $a \in N^{\tau}$ and so Definition 3.3(i) holds, whence $\left(N^{\tau}, \tau\right)$ is a pseudo right congruence pair.

Now let $\rho$ be a right congruence with $\operatorname{tr} \rho=\tau$. Let $K=\operatorname{ker} \rho$. By Theorem 3.5, $(K, \tau)$ is a right congruence pair with $\rho=\rho_{(K, \tau)}$. Let $a \in K$. Then by Definition 3.3(ii), we have $a \in N^{\tau}$ whence $K \subseteq N^{\tau}$. By Proposition 3.4 we have $\rho \subseteq \rho_{\left(N^{\tau}, \tau\right)}$.

We remark that in general, $\left(N^{\tau}, \tau\right)$ is not a right congruence pair (that is to say, in general $\left.\operatorname{ker} \rho_{\left(N^{\tau}, \tau\right)} \subsetneq N^{\tau}\right)$. For example, let $S$ be the orthogonal sum of two copies of $\mathbf{B}_{2}$, say $S=\left\{0, a, a^{-1}, a a^{-1}, a^{-1} a, b, b^{-1}, b b^{-1}, b^{-1} b\right\}$ and let $\rho$ be the Rees right congruence on $S$ modulo $\left\{0, a, a a^{-1}\right\}$, a right ideal of $S$. Then $b \in N^{\operatorname{tr} \rho}$ but $b \notin \operatorname{ker} \rho$.

Proposition 4.2. Let $\tau \in \mathscr{C}(E)$, and let

$$
N_{\tau}=\left\{a \in S \mid a a^{-1} \tau \text { e for some } e \in E \text { with } e a=e\right\} .
$$

Then $\left(N_{\tau}, \tau\right)$ is a right congruence pair for $S$ and $\rho_{\left(N_{\tau}, \tau\right)}$ is the least right congruence on $S$ with trace $\tau$.

Proof. It is obvious that $E \subseteq N_{\tau}$. Let $a, b \in N_{\tau}$. Then there exist $e, f \in E$ with $a a^{-1} \tau e, b b^{-1} \tau f$ and $e a=e, f b=f$. Thus

$$
\begin{aligned}
(a b)(a b)^{-1} & =a a^{-1}(a b)(a b)^{-1} \tau e(a b)(a b)^{-1} \\
& =(e a) b b^{-1} a^{-1} e=e b b^{-1} \tau e f
\end{aligned}
$$

and $(e f)(a b)=f(e a) b=f e b=e f$ whence $a b \in N_{\tau}$.

Next, let $a \geq b \in N_{\tau}$ with $a a^{-1} \tau b b^{-1}$. Then there exists $e \in E$ with $b b^{-1} \tau e=e b$. Since $a \geq b$ we have $b b^{-1} a=b$ and so $\left(e b b^{-1}\right) a=e b=e=$ $e b b^{-1}$, while $a a^{-1} \tau b b^{-1} \tau e=e b b^{-1}$. Thus $f=e b b^{-1}$ satisfies $a a^{-1} \tau f=$ $f a$ and so $a \in N_{\tau}$. Thus Definition 3.3(i) holds.

Now suppose that $a \in N_{\tau}$. Then $a a^{-1} \tau e^{\prime}=e^{\prime} a$ for some $e^{\prime} \in E$ and so for any idempotent $e$ we have $a e a^{-1}=a a^{-1} a e a^{-1} \tau e^{\prime} a e a^{-1} e^{\prime}=e^{\prime} e$. Thus if $e, f \in E$ and $e \tau f$, then $a e a^{-1} \tau e^{\prime} e \tau e^{\prime} f \tau a f a^{-1}$ and so Definition 3.3(ii) holds. Consequently $\left(N_{\tau}, \tau\right)$ is a pseudo right congruence pair for $S$.

Now let $\rho$ be any right congruence with trace $\tau$. Let $a \in N_{\tau}$ and $e \in E$ with $a a^{-1} \tau e=e a$. Then $a a^{-1} \rho e$ whence $a \rho e a=e$ and so $a \in \operatorname{ker} \rho$. Thus $N_{\tau} \subseteq \operatorname{ker} \rho$. In particular, $N_{\tau} \subseteq \operatorname{ker} \rho_{\left(N_{\tau}, \tau\right)}$ and so by Proposition 3.4 we have $N_{\tau}=\operatorname{ker} \rho_{\left(N_{\tau}, \tau\right)}$ whence $\left(N_{\tau}, \tau\right)$ is a right congruence pair. Now by Theorem 3.5, $\rho=\rho_{(K, \tau)}$ and then by Proposition 3.4 we obtain $\rho_{\left(N_{\tau}, \tau\right)} \subseteq \rho$. It follows that $\rho_{\left(N_{\tau}, \tau\right)}$ is the least right congruence with trace $\tau$.

We turn our attention now to the problem of determining which full subsemigroups of $S$ arise as kernels of right congruences. It is interesting to observe 
as a result of Lemma 3.10(i) that every full subsemigroup $K$ of $S$ is a pseudo right kernel of some right congruence on $S$, namely $\rho_{(K, \varepsilon)}$. Thus the pseudo right kernels of $S$ turn out to be just the full subsemigroups of $S$.

Definition 4.3. A subset $K$ of $S$ is a right kernel in $S$ if

(i) $K$ is a full subsemigroup of $S$,

(ii) for each $a \in K$, there exists $b \leq a$ such that $b^{-1} \in K$, and $b x \in K$ implies $a x \in K$ for any $x \in S$.

Our goal is to show that a subset $K$ of $S$ is a right kernel in $S$ if and only if $K$ is the kernel of some right congruence on $S$. Note that if $K=\operatorname{ker} \rho$ for some right congruence $\rho$, then $\rho \subseteq \mathrm{P}_{K}$.

Proposition 4.4. The following conditions on a subset $K$ of $S$ are equivalent.

(i) $K$ is a right kernel in $S$.

(ii) $K=\operatorname{ker} \rho$ for some $\rho \in \mathscr{R} \mathscr{C}(S)$.

(iii) $K=\operatorname{ker}_{K}$.

Proof. If $K=\operatorname{ker} \rho$ for some $\rho \in \mathscr{R} \mathscr{C}(S)$, then $K \subseteq \operatorname{ker} \mathrm{P}_{K}$ and $K$ is saturated by $\mathrm{P}_{K}$ and so $K=\operatorname{ker} \mathrm{P}_{K}$. Thus (ii) and (iii) are equivalent. We show that (i) and (iii) are equivalent. Evidently, Definition 4.3(i) is a necessary condition for each of (i) and (iii). We show that for a full subsemigroup $K$ of $S$,

$$
\begin{aligned}
& \operatorname{ker} \mathbf{P}_{K}=\left\{a \in K \mid \text { there exists } b \leq a \text { such that } b^{-1} \in K,\right. \\
& \text { and } b x \in K \text { implies } a x \in K \text { for any } x \in S\}
\end{aligned}
$$

whence the result follows. Let $K$ be a full subsemigroup of $S$ and let $a \in$ ker $\mathrm{P}_{K}$, so that $a \mathrm{P}_{K} e$ for some $e \in E$. Then $a e \mathrm{P}_{K} a$ and so for all $x \in S$, $a x \in K$ if and only if $a e x \in K$. Let $b=a e$. Then $b \leq a$ and since $a a^{-1} \in K$ we obtain $b^{-1}=e a^{-1} \in K$. Conversely, suppose that $a \in K$ is such that there exists $b \leq a$ with $b^{-1} \in K$ and $b x \in K$ implies that $a x \in K$ for all $x \in S$. We show that $a \mathrm{P}_{K} e=b^{-1} b$ whence $a \in \operatorname{ker}_{K}$. By definition of $\mathrm{P}_{K}$, we must show that for all $x \in S, a x \in K$ if and only if $e x \in K$. Let $x \in S$, and assume that $a x \in K$. Then $b x=b b^{-1} a x \in K$, so that $e x \in K$ since $b^{-1} \in K$. On the other hand, if $e x \in K$, then since $a \in K$ we obtain $a e x \in K$. But $a b^{-1}=b b^{-1}$ and so $b x=a b^{-1} b x=a e x \in K$ and so by hypothesis we have $a x \in K$, as required.

\section{KERNEL CLASSES}

The function $\rho \mapsto \operatorname{ker} \rho \quad(\rho \in \mathscr{R} \mathscr{C}(S))$, called the kernel map, maps $\mathscr{R} \mathscr{C}(S)$ onto the set of right kernels in $S$. The classes of the induced equivalence relation on $\mathscr{R} \mathscr{C}(S)$ are called the kernel classes of $S$. The restriction of the kernel mapping to the sublattice $\mathscr{C}(S)$ of $\mathscr{R} \mathscr{C}(S)$ is a complete $\mathrm{n}$-homomorphism of $\mathscr{C}(S)$ onto the set of kernels in $S$ (see [9, III.4.8]) and the equivalence classes of $\mathscr{C}(S)$ under the induced equivalence relation are intervals. It is also known (see [9, III.4.11]) that the restricted map is not $\vee$-preserving.

It turns out that the kernel map on right congruences does not preserve even finite intersections in general. The problem of course stems from the fact that an element $a$ might be in the kernel of a right congruence, yet not be related 
to $a a^{-1}$. It is obvious that if $S$ is an inverse semigroup for which every right congruence $\rho$ has the property that $a \in \operatorname{ker} \rho$ if and only if $a \rho a a^{-1}$, then the kernel map in that case is a complete $\cap$-homomorphism from $\mathscr{R} \mathscr{C}(S)$ onto the set of right kernels (see $\S 8$ ).

To illustrate the situation, consider the following example. Let $\mathbf{B}_{3}$ denote the combinatorial Brandt semigroup with three nonzero idempotents. Then if $a \in \mathbf{B}_{3}^{*}$, we see that $K=E \cup \mathbf{R}_{a}$ and $L=E \cup\left\{a, a a^{-1}, a^{-1} a, a^{-1}\right\}$ are right kernels in $\mathbf{B}_{3}$ but $K \cap L$ is not a right kernel in $\mathbf{B}_{3}$.

The kernel class of a right kernel $K$ has a greatest element, namely $\mathrm{P}_{K}$ whence each kernel class is closed under join, just as for congruences. However, the following example demonstrates that in general, kernel classes are not closed under intersection, whence in general, there is no least element for a kernel class.

In [8, Proposition 6.1], Nico describes all one-sided congruences on the bicyclic semigroup $\mathscr{B}$. Let $a, b$ be generators of $\mathscr{B}$ with $a b=1$. Let $\alpha$ be the right congruence given by $b^{m} a^{n} \alpha b^{p} a^{q}$ if and only either $b^{m} a^{n}=b^{p} a^{q}$ or $n=q$ and $m, p \geq 1$. Now let $\beta$ be the right congruence given by $b^{m} a^{n} \beta b^{p} a^{q}$ if and only if $b^{m} a^{n}=b^{p} a^{q}$ or $m, p \leq 1$ and $n-m=q-p$. Then for any $b^{m} a^{n} \in \mathscr{B}$, we have

$$
\left(b^{m} a^{n}\right) \alpha= \begin{cases}\left\{a^{n}\right\} & \text { if } m=0, \\ L_{\left(b^{m} a^{n}\right)} \backslash\left\{a^{n}\right\} & \text { if } m>0,\end{cases}
$$

while the $\beta$-classes are either singletons or of the form $\left\{a^{n}, b a^{n+1}\right\}$ for $n \geq 0$. Thus if we set $\rho=\alpha \vee \beta$, then $\rho$ is a right congruence with kernel $\mathscr{B}$. As well, Green's relation $\mathscr{L}$ is a right congruence with kernel $\mathscr{B}$. However, $\mathscr{L} \cap \rho=\alpha$ and $\operatorname{ker} \alpha=(\mathscr{B} \backslash R 1) \cup\{1\}$.

This example demonstrates why one cannot conclude from Proposition 3.8 that the kernel mapping is a $\cap$-homomorphism. The pair $(\mathscr{B}, \operatorname{tr}(\mathscr{L} \cap \rho))$ is a pseudo right congruence pair which determines $\mathscr{L} \cap \rho$ but it is not a right congruence pair.

We remark that if $K$ is a full inverse subsemigroup of $S$, then by Lemma 3.10(ii), $(K, \varepsilon)$ is a right congruence pair and so the kernel class of $K$ is an interval, namely $\left[\rho_{(K, \varepsilon)}, \mathrm{P}_{K}\right]$.

\section{Trace Classes}

Motivated by the known results for the trace function on the congruence lattice of an inverse semigroup (see [9, III.2.5]), we define a mapping $\rho \mapsto$ tr $\rho$ for $\rho \in \mathscr{R} \mathscr{C}(S)$, the lattice of right congruences on $S$. This mapping is called the trace map on $\mathscr{R} \mathscr{C}(S)$. The equivalence classes of the equivalence relation induced on $\mathscr{R} \mathscr{C}(S)$ by the trace map are called the trace classes. By Propositions 4.1 and 4.2 , trace classes are intervals in $\mathscr{R} \mathscr{C}(S)$. Recall the definitions of $N_{\tau}$ and $N^{\tau}$ from Propositions 4.1 and 4.2.

Definition 6.1. For any $\tau \in \mathscr{C}(E)$, let $\tau_{\text {rmin }}=\rho_{\left(N_{\tau}, \tau\right)}$ and $\tau_{r \max }=\rho_{\left(N^{\tau}, \tau\right)}$.

Proposition 6.2. The trace map is a complete $\cap$-homomorphism of $\mathscr{R} \mathscr{C}(S)$ onto $\mathscr{C}(E)$ and for any $\rho \in \mathscr{R} \mathscr{C}(S)$, the trace class of $\rho$ is the interval $\left[(\operatorname{tr} \rho)_{\text {rmin }},(\operatorname{tr} \rho)_{\text {rmax }}\right]$.

Proof. That the trace map is a complete $\mathrm{n}$-homomorphism follows immediately from Proposition 3.9. The description of the trace class of a right congruence is essentially Theorem 3.1 of [7]. 
We remark here that while the trace classes do form complete sublattices of $\mathscr{R} \mathscr{C}(S)$, they fail to be modular sublattices, in contrast with the situation for congruences (see for example [9, III.2.5]). In fact, Jones [5, 6] studied inverse semigroups for which the trace class of $\varepsilon$ is semimodular, modular or distributive. It was shown for example in [5, Corollaries 3.5 and 3.6] that every combinatorial Brandt semigroup has semimodular trace class of $\varepsilon$ while every combinatorial Brandt semigroup with more than four idempotents has nonmodular trace class of $\varepsilon$.

Another important difference between congruences and right congruences on $S$ is that the trace map on right congruences is not a $V$-homomorphism. An example is given in [10] of two right congruences on a Brandt semigroup having universal join, yet one right congruence is idempotent separating and the other has exactly one nontrivial trace class, and that class has just two elements.

Yet another difference occurs in the interplay between the congruence lattice structure and the max operator. This can be illustrated with the aid of the following result.

Lemma 6.3. Let $\tau \in \mathscr{C}(E)$. Then $\tau$ is normal if and only if $\operatorname{ker} \tau_{\text {rmax }}=S$.

Proof. Suppose that $\tau$ is normal. It is immediate from the definition of $N^{\tau}$ that $N^{\tau}=S$. This fact together with Proposition 3.4 implies that $\operatorname{ker} \tau_{\text {rmax }}=S$. Conversely, if $\operatorname{ker} \tau_{\text {rmax }}=S$, then since $\operatorname{tr} \tau_{\text {rmax }}=\tau$, we see that $(S, \tau)$ is a right congruence pair whence by Definition 3.3 it follows that $\tau$ is normal.

We may now demonstrate that $\tau \subseteq \tau^{\prime}$ does not imply that $\tau_{r \max } \subseteq \tau_{\max }^{\prime r}$, unlike the situation for congruences on an inverse semigroup. For example, if $\tau=\varepsilon$ and $\tau^{\prime}$ is a nonnormal congruence on $E$, then $\tau \subseteq \tau^{\prime}$ while $\operatorname{ker} \tau_{\text {rmax }}=S$ and $\operatorname{ker} \tau_{\text {max }}^{\prime r} \neq S$ and so we conclude that $\tau_{\text {rmax }} \nsubseteq \tau_{\text {max }}^{\prime r}$.

Normality of a congruence $\tau$ can also be described in terms of $\tau_{\text {rmin }}$. Obviously, if $\tau_{\text {rmin }}$ is a congruence, then $\tau$ is normal. That the converse is also true is a consequence of the next result.

Proposition 6.4. Let $\tau$ be a congruence on $E$, and let

$$
N(\tau)=\left\{a \in S \mid e \tau f \text { implies aea } a^{-1} \tau \text { afa } a^{-1} \text { and } a^{-1} e a \tau a^{-1} f a\right\} .
$$

Then $N(\tau)$ is the greatest full inverse subsemigroup of $S$ with respect to which $\tau$ is normal, and $\left.\tau_{r \min }\right|_{N(\tau)}$ is a congruence on $N(\tau)$.

Proof. It is evident that $N(\tau)$ is the greatest inverse subsemigroup of $S$ contained in $N^{\tau}$ and that $N(\tau)$ must therefore be the greatest inverse subsemigroup of $S$ with respect to which $\tau$ is normal. It remains to show that $\left.\tau_{r \min }\right|_{N(\tau)}$ is a congruence on $N(\tau)$. Let $\tau_{\text {min }}$ denote the least congruence on $N(\tau)$ with trace the normal congruence $\tau$. Since $\operatorname{tr}\left(\left.\tau_{r \min }\right|_{N(\tau)}\right)=\tau=\operatorname{tr} \tau_{\min }$, it follows from Theorem 3.5 that if $\operatorname{ker}\left(\left.\tau_{r \min }\right|_{N(\tau)}\right)=\operatorname{ker} \tau_{\min }$, then $\left.\tau_{r \min }\right|_{N(\tau)}=\tau_{\min }$. Let $a \in \operatorname{ker} \tau_{\min }$. Then (see [9, III.2.4]) there exists $e \in E$ with $e a=e a a^{-1}$ and $e \tau a a^{-1}$. Let $f=e a a^{-1}$. We have $f a=f$ and $f \tau a a^{-1}$ whence $a \in \operatorname{ker}\left(\left.\tau_{r \min }\right|_{N(\tau)}\right)$. Conversely, suppose that $a \in \operatorname{ker}\left(\left.\tau_{r \min }\right|_{N(\tau)}\right)$. Then for some $f \leq a^{-1} a$ we have $a \tau_{r \text { min }} f$ whence $a f, f a^{-1} \in N_{\tau}$ and $a f a^{-1} \tau a a^{-1}$, $f a^{-1} a=f$. Let $e=a f a^{-1}$. Then $e a=a f \in N_{\tau}$ whence there exists $e^{\prime} \in E$ with $e^{\prime} e a=e^{\prime}$ and $e=e a a^{-1} \tau e^{\prime}$. Thus $e^{\prime \prime}=e e^{\prime}$ satisfies $e^{\prime \prime} a=e^{\prime \prime}$ and $e^{\prime \prime} \tau e=a f a^{-1} \tau a a^{-1}$, whence $a \tau_{\min } a a^{-1}$ and so $a \in \operatorname{ker} \tau_{\min }$. We have $\operatorname{ker}\left(\left.\tau_{r \min }\right|_{N(\tau)}\right)=\operatorname{ker} \tau_{\min }$, as required. 
Corollary 6.5. Let $\tau$ be a congruence on $E$. Then $\tau$ is normal if and only if $\tau_{\text {rmin }}$ is a congruence.

\section{RIGHT KERNEL SYSTEMS}

Each congruence on an inverse or even regular semigroup is completely determined by its idempotent classes. Specifically, on any semigroup each congruence can be written as the intersection of the principal congruences on the different congruence classes. For a regular semigroup, it is fairly straightforward to show that each congruence is the intersection of the principal congruences on just the idempotent congruence classes. The notion of specifying a congruence on an inverse semigroup by giving its idempotent congruence classes was introduced by Preston [12]. He gave the name kernel normal system to a collection of subsets of an inverse semigroup which constituted the idempotent congruence classes for some congruence, and he presented an independent set of axioms that a collection must satisfy in order to be a kernel normal system. In [7], Meakin has carried out the same program for right congruences on an inverse semigroup.

Definition 7.1 (see [7, Definition 2.1]). A collection $\mathscr{A}=\left\{A_{\alpha} \mid \alpha \in \Lambda\right\}$ of subsets of $S$ is called a right kernel system of $S$ if it satisfies the following conditions:

(i) $A_{\alpha} \cap A_{\beta}=\varnothing$ for $\alpha, \beta \in \Lambda, \alpha \neq \beta$,

(ii) $E \subseteq K=\bigcup_{\alpha \in \Lambda} A_{\alpha}$,

(iii) $E \cap A_{\alpha} \neq \varnothing$ for $\alpha \in \Lambda$,

(iv) for each $a \in K$ and $\alpha \in \Lambda$, there exists $\beta \in \Lambda$ such that $A_{\alpha} a \subseteq A_{\beta}$,

(v) for each $a \in S, a \in A_{\alpha}$ and $a a^{-1} \in A_{\beta}$ implies that $A_{\alpha} a^{-1} \subseteq A_{\beta}$,

(vi) for $a \in S, \beta \in \Lambda$ with $a a^{-1} \in A_{\beta}$, if $K a^{-1} \cap A_{\beta} \neq \varnothing$ then $a \in K$.

Meakin [7, Theorem 2.1] shows that $\mathscr{A}$ is a right kernel system of $S$ if and only if $\mathscr{A}$ is the set of idempotent classes of a right congruence on $S$. We offer another set of axioms which characterize right kernel systems. Depending on the problem under study, this list may be more convenient to use than that given in Definition 7.1. This alternative axiom set amounts to requiring that each $A_{\alpha}$ be a congruence class of the principal right congruence on $A_{\alpha}$, and for each $A_{\beta}$ in the collection, $A_{\alpha}$ should be contained in a class of the principal right congruence on $A_{\beta}$.

Proposition 7.2 (cf. [1, Theorem 10.4]). Let $\mathscr{A}=\left\{A_{\alpha} \mid \alpha \in \Lambda\right\}$ be a collection of subsets of $S$. Then $\mathscr{A}$ is a right kernel system if and only if $\mathscr{A}$ satisfies (i), (ii), (iii) of Definition 7.1 and the following condition:

(vii) for all $x \in S, \alpha, \beta \in \Lambda, A_{\alpha} x \cap A_{\beta} \neq \varnothing$ implies that $A_{\alpha} x \subseteq A_{\beta}$.

Proof. It follows from [7, Theorem 2.1] that if $\mathscr{A}$ is a right kernel system, then (vii) holds. For the converse, it is sufficient to show that there is a right congruence $\rho$ for which $\mathscr{A}=\{e \rho \mid e \in E\}$. Let $\rho=\bigcap_{\alpha \in \Lambda} \mathrm{P}_{\alpha}$, where for convenience we denote the principal right congruence on $A_{\alpha}$ by $\mathrm{P}_{\alpha}$. For $\beta \in$ $\Lambda$, choose $e \in A_{\beta} \cap E$ and suppose that $a \rho e$. Then for all $x \in S$ and $\alpha \in \Lambda, a x \in A_{\alpha}$ if and only if $e x \in A_{\alpha}$. Since $e \in A_{\beta}$, it follows that $a \in A_{\beta}$ whence $e \rho \subseteq A_{\beta}$. Conversely, let $a \in A_{\beta}$. For $x \in S$, if $A_{\beta} x \cap A_{\alpha} \neq \varnothing$, 
then $A_{\beta} x \subseteq A_{\alpha}$ whence $a x \in A_{\alpha}$ if and only if $e x \in A_{\alpha}$. Thus $a \mathrm{P}_{\alpha} e$ for all $\alpha \in \Lambda$ and so $a \rho e$. Thus $A_{\beta} \subseteq e \rho$. This establishes that $\mathscr{A}$ is the collection of idempotent classes of $\rho$.

We conclude with an example showing that a right congruence on a regular semigroup need not be determined by its idempotent classes. Construct a four element orthodox completely regular semigroup as the direct product of the cyclic group of order two and a two element right zero semigroup. The equivalence relation whose only nontrivial class contains the two nonidempotent elements is a right congruence each of whose idempotent classes is a singleton, as is the identity relation.

\section{Right CONGRUENCES ON A ClifFord SEMIGRouP}

Recall first that Clifford semigroup is a synonym for semilattice of groups. In a Clifford semigroup $S$, we have $a a^{-1}=a^{-1} a$ for all $a \in S$. We shall write $a^{0}=a^{-1} a$ for convenience.

Proposition 8.1. The following statements hold for any Clifford semigroup $S$.

(i) For $\rho \in \mathscr{R} \mathscr{C}(S)$ and $a, b \in S$, we have

$$
a \rho b \Leftrightarrow a b^{0} \rho b a^{0}, a^{0} \rho b^{0}
$$

and $\operatorname{ker} \rho=\left\{a \in S \mid a \rho a^{0}\right\}$.

(ii) A pair $(K, \tau)$ is a right congruence pair for $S$ if and only if

$(\alpha) K$ is a full inverse subsemigroup of $S$,

( $\beta) \tau$ is a congruence on $E$,

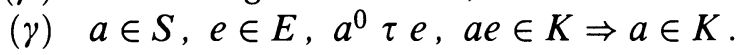

In such a case, for any $a, b \in S$, we have

$$
a \rho_{(K, \tau)} b \Leftrightarrow a b^{-1} \in K, a^{0} \tau b^{0} .
$$

(iii) $A$ subset $K$ of $S$ is a right kernel in $S$ if and only if $K$ is a full inverse subsemigroup of $S$.

(iv) If $\tau \in \mathscr{C}(E)$, then $\tau_{\text {rmin }}=\tau_{\min }$ and $\tau_{\text {rmax }}=\tau_{\max }$

Proof. (i) If $a \rho b$, then $a b^{0} \rho b b^{0}=b \rho a=a a^{0} \rho b a^{0}$,

$$
a^{0}=a a^{-1} \rho b a^{-1}=\left(b a^{0}\right) a^{-1} \rho\left(a b^{0}\right) a^{-1}=a^{0} b^{0}
$$

and analogously $b^{0} \rho b^{0} a^{0}$ so that $a^{0} \rho b^{0}$. Conversely, if $a b^{0} \rho b a^{0}$ and $a^{0} \rho b^{0}$, then

$$
a=a^{0} a \rho b^{0} a=a b^{0} \rho b a^{0}=a^{0} b \rho b^{0} b=b .
$$

If $a \rho e$, where $e \in E$, then

$$
a=a a^{0} \rho e a^{0}=a e a^{-1} \rho e a^{-1} \rho a a^{-1}=a^{0} .
$$

(ii) Let $(K, \tau)$ be a right congruence pair for $S$. By Theorem 3.5, there exists $\rho \in \mathscr{R} \mathscr{C}(S)$ such that $K=\operatorname{ker} \rho$ and $\tau=\operatorname{tr} \rho$. Let $a \in K$. Then by part (i), $a \rho a^{-1} a$ and so $a a^{-1} \rho a^{-1}$. Thus $K$ is a full inverse subsemigroup of $S$. This establishes $(\alpha)$; item $(\beta)$ is obvious. Let $a \in S, e \in E, a^{0} \tau e$ 
and $a e \in K$. Then $a=a^{0} a \rho e a=a e \rho(a e)^{0}$ and thus $a \in K$, whence $(\gamma)$ holds.

Conversely, assume that $(\alpha),(\beta)$ and $(\gamma)$ hold. If the conditions of Definition 3.3(i) hold, then with $e=b^{0}$ we obtain $a \in K$ by $(\gamma)$. Hence Definition 3.3(i) is satisfied; part (ii) holds trivially; part (iii) holds since $K$ is closed under inverses. Therefore $(K, \tau)$ is a right congruence pair for $S$.

Now suppose that $(K, \tau)$ is a right congruence pair for $S$ and let $a, b \in S$. By part (i), it suffices to prove that $a b^{-1} \rho\left(a b^{-1}\right)^{0}$ if and only if $a b^{0} \rho b a^{0}$, which follows easily.

(iii) Let $K$ be a right kernel in $S$. Then by Proposition $4.4, K=\operatorname{ker} \rho$ for some $\rho \in \mathscr{R} \mathscr{C}(S)$. Now by part (ii) $(\alpha)$ we have that $K$ is a full inverse subsemigroup of $S$. Conversely, if $K$ is a full inverse subsemigroup of $S$, then by Lemma $3.10(\mathrm{i})$ it is the kernel of a right congruence on $S$.

(iv) Let $\tau \in \mathscr{C}(E)$. By Corollary 5.5, we know that $\tau_{r m i n}$ is a congruence on $S$ since $\tau$ is normal in $S$, whence $\tau_{\text {rmin }}=\tau_{\text {min }}$.

Finally, since $\tau_{\max } \subseteq \tau_{\text {rmax }}$ always holds, it suffices to show that $\tau_{\text {rmax }} \subseteq$ $\tau_{\text {max }}$. Let $a \tau_{\text {rmax }} b$. By part (i), we have $a^{0} \tau_{\text {rmax }} b^{0}$ and thus $a^{0} \tau b^{0}$. But then for every $e \in E$, we have $a e a^{-1} \tau b e b^{-1}$ and so $a \tau_{\max } b$ (see [9, III.2.4]).

We note that Clifford semigroups whose right congruence lattices satisfied certain conditions were discussed in [3].

\section{All RIGHT CONGRUENCES ARE TWO-SIDED}

We characterize here all inverse semigroups for which every right congruence is two-sided. This is the semigroup analogue of the condition on a group that every subgroup be normal. Note that for a relation $\rho$ on $S$, the relation $\rho^{\text {inv }}$ defined by $a \rho^{\text {inv }} b$ if $a^{-1} \rho b^{-1}$ is a left congruence on $S$ if and only if $\rho$ is a right congruence on $S$. Thus the requirement that every right congruence be two-sided is equivalent to the requirement that every one-sided congruence be two-sided.

For any nonempty subset $X$ of $S$, let $\rho_{X}$ denote the right congruence on $S$ defined by $a \rho_{X} b$ if $X a=X b$.

Lemma 9.1. Let $e \in E$ and let $H$ be a subgroup of $H_{e}$. The right congruence $\rho_{H}$ is idempotent separating if and only if $S$ is a monoid and $e$ is the identity of $S$.

Proof. Suppose that $\rho_{H}$ is idempotent separating. Since $x \rho_{H}$ ex for every $x \in S$, we obtain that $f=e f$ for every $f \in E$. Thus $S$ has identity $e$. Conversely, suppose that $S$ is a monoid and that $e$ is the identity of $S$. If $a \rho_{H} b$, then $S a=S H a=S H a b=S b$ and so $a \mathscr{L} b$. It follows that $\rho_{H} \subseteq$ $\mathscr{L}$ whence $\rho_{H}$ is idempotent-separating.

Lemma 9.2. If $S$ is an inverse semigroup for which every nonidempotent separating right congruence is two-sided, then $S$ is a Clifford semigroup.

Proof. Let $e \in E$ and let $\rho_{e}$ denote the right congruence $\rho_{\{e\}}$. If $\rho_{e}$ is idempotent separating, then by Lemma $9.1 e$ must be the identity of $S$. Otherwise, $\rho_{e}$ is not idempotent separating and so by hypothesis, $\rho_{e}$ is two-sided. But then 
$x \rho_{e} e x$ implies that $x^{-1} \rho_{e} x^{-1} e$ and so $e x^{-1}=e x^{-1} e$ for every $x \in S$. But then $e x=e x e$ for every $x \in S$ whence $x^{-1} e=(e x)^{-1}=e x^{-1} e=e x^{-1}$ for every $x \in S$. Thus $e$ is central and so $S$ is a Clifford semigroup.

A non-Abelian group is said to be Hamiltonian if each of its subgroups is normal. A Hamiltonian group is the direct product of a quaternion group with an Abelian group in which every element is of finite order and an Abelian group of exponent two (see for example [4, Theorem 12.5.4]).

Proposition 9.3. If $S$ is an inverse semigroup with $|E(S)| \geq 3$, then every nonidempotent separating right congruence on $S$ is two-sided if and only if $S$ is a Clifford semigroup in which every maximal subgroup is either Abelian or Hamiltonian.

Proof. Suppose first that every nonidempotent separating right congruence on $S$ is two-sided. By Lemma 9.1, $S$ is a Clifford semigroup. Let $e \in E$ and let $G_{e}$ denote the $\mathscr{H}$-class of $e$. Let $H$ be a subgroup of $G_{e}$. Assume that $e$ is not the identity of $S$. Then by Lemma 9.1 we see that $\rho=\rho_{H}$ is not idempotent separating and so by hypothesis $\rho$ is a two-sided congruence on $S$. But then $\left.\rho\right|_{G_{e}}$ is a congruence on $G_{e}$ and so $H$ is normal in $G_{e}$. Now assume that $e$ is the identity of $S$ and consider the principal right congruence $\mathrm{P}_{H}$ on $S$. Since the Rees right congruence modulo $S \backslash G_{e}$ saturates $H$, if follows that $S \backslash G_{e}$ is a $\mathrm{P}_{H}$-class. Moreover, since $|E(S)| \geq 3$, we see that $S \backslash G_{e}$ contains at least two idempotents and so $\mathrm{P}_{H}$ does not separate idempotents. Thus $\mathrm{P}_{H}$ is two-sided and so $\left.\mathrm{P}_{H}\right|_{G_{e}}$ is a congruence on $G_{e}$ whose idempotent congruence class is $H$, whence $H$ is normal in $G_{e}$.

Conversely, suppose that $S$ is a Clifford semigroup in which every maximal subgroup is either Abelian or Hamiltonian. By Proposition 8.1(ii) and [9, III.1.3], a right congruence pair $(K, \tau)$ for $S$ is a congruence pair if and only if $K$ is self-conjugate. But every inverse subsemigroup of $S$ is self-conjugate and so every right congruence on $S$ is two-sided.

We remark that the nonidempotent separating right congruences do not provide enough information about the subgroups of $S$ when $S$ has too few idempotents. This is obviously true when $S$ is a group. If $S$ is a chain of two groups with trivial structure homomorphism, then any right congruence which identifies the two idempotents of $S$ also identifies the top group with those idempotents and so the subgroup structure of the top group is hidden from us if we are only permitted to use nonidempotent separating right congruences.

Corollary 9.4. Every right congruence on an inverse semigroup $S$ is two-sided if and only if $S$ is a Clifford semigroup in which every maximal subgroup is either Abelian or Hamiltonian.

Proof. For the direct part, the only case to consider is that when $|E(S)|=2$. The proof of Proposition 9.3 can be used since every right congruence on $S$, idempotent separating or not, is two-sided.

The proof of the converse is the same as that of Proposition 9.3.

Corollary 9.5. An inverse semigroup $S$ has no proper right congruences if and only if $S$ is either a finite cyclic group of prime order or a semilattice of order 1 or 2 . 


\section{RIGHT CONGRUENCES FORM A CHAIN}

We determine here all inverse semigroups whose right congruences are linearly ordered under inclusion. As we shall see, this is a very strong condition indeed, for right congruences are abundant in almost every inverse semigroup. It was shown in [7] that the lattice of idempotent separating right congruences on $S$ is isomorphic to the lattice of full inverse subsemigroups of $S$ and we have classified in [11] the inverse semigroups in which the full inverse subsemigroups form a chain.

Proposition 10.1. The lattice of right congruences on an inverse semigroup $S$ is a chain if and only if $S$ is isomorphic to a subsemigroup of $\left(Z_{p \infty}\right)^{0}$ for some prime $p$.

Proof. Suppose first of all that $\mathscr{R} \mathscr{C}(S)$ is a chain. For $e \in E$, let $\rho_{e}$ denote the right congruence on $S$ given by $a \rho_{e} b$ if $e a=e b$. Let $e, f \in E$. Then $\rho_{e}$ and $\rho_{f}$ are comparable. Assume that $\rho_{e} \subseteq \rho_{f}$. Then from $f \rho_{e}$ ef we obtain $f \rho_{f} e f$ whence $f=e f$. It follows that $E$ is a chain. But now by [11, IV.2.1], the subsemigroup of $S$ generated by the nonidempotents of $S$ is a direct product of a chain $Y$ and a group $G$, where $G$ is a subgroup of $\mathrm{Z}_{p^{\infty}}$ for some prime $p$. As a consequence, $S$ is a Clifford semigroup. Now for a Clifford semigroup, every congruence on $E$ is a normal congruence and so is the trace of a congruence on $S$, whence $\mathscr{C}(E)$ must be a chain. This forces $|E| \leq 2$. If $|E|=1$, then $S$ is isomorphic to a subgroup of $Z_{p \infty}$ for some prime $p$. Suppose that $|E|=2$. Then there is a retraction of $S$ onto the bottom group. Let $\lambda$ denote the congruence induced by this retraction. Then $\lambda$ identifies the two idempotents of $S$ and so $\lambda \nsubseteq \rho$, the Rees right congruence of $S$ modulo the bottom group. By hypothesis we have $\rho \subseteq \lambda$ whence the bottom group is a singleton. Thus $S$ is a group with zero. Either the group is trivial or else, as described above, it is a subgroup of $\mathrm{Z}_{p^{\infty}}$. In any event, $S$ is isomorphic to a subsemigroup of $\left(\mathrm{Z}_{p^{\infty}}\right)^{0}$.

The converse is immediate.

\section{REFERENCES}

1. A. H. Clifford and G. B. Preston, The algebraic theory of semigroups, Vol. II, Amer. Math. Soc., Providence, R. I., 1967.

2. G. Duchamp, Étude du treillis des congruences à droite, Semigroup Forum 33 (1986), 31-46.

3. J. B. Fountain and P. Lockley, Semilattices of groups with distributive congruence lattices, Semigroup Forum 14 (1977), 81-91.

4. M. Hall, The theory of groups, Macmillan, New York, 1959.

5. P. R. Jones, Semimodular inverse semigroups, J. London Math. Soc. 17 (1978), 446-456.

6. __ Distributive inverse semigroups, J. London Math. Soc. 17 (1978), 457-466.

7. J. Meakin, One-sided congruences on inverse semigroups, Trans. Amer. Math. Soc. 206 (1975), 67-82.

8. W. R. Nico, A classification of indecomposable S-sets, J. Algebra 54 (1978), 260-272.

9. M. Petrich, Inverse semigroups, Wiley-Interscience, New York, 1984.

10. M. Petrich and S. A. Rankin, Right congruences on a Brandt semigroup, preprint.

11. _ A classification of inverse semigroups whose full inverse subsemigroups form a chain, preprint. 
12. G. B. Preston, Inverse semigroups, J. London Math. Soc. 29 (1954), 396-403.

13. H. E. Scheiblich, Kernels of inverse semigroup homomorphisms, J. Austral. Math. Soc. 18 (1974), 289-292.

14. V. V. Wagner, The theory of generalized heaps and generalized groups, Mat. Sb. 32 (1953), 545-632. (Russian)

Department of Mathematics, University of Western Ontario, London, Canada 